\title{
The three-dimensional positioning system at the VINETA.II experiment - a multipurpose tool for in situ plasma diagnostics
}

\author{
Ilya Shesterikov, ${ }^{a, 1}$ Dusan Milojevic, ${ }^{a}$ Adrian von Stechow, ${ }^{a}$ Kian Rahbarnia, ${ }^{a}$ Olaf Grulke ${ }^{a, 2}$ \\ and Thomas Klinger ${ }^{a, 3}$ \\ ${ }^{a}$ Max Planck Institute for Plasma Physics, 17491, Greifswald, Germany \\ E-mail: ilys@ipp.mpg.de
}

ABSTRACT: The manipulator systems installed at the Vineta.II magnetic reconnection experiment are essential elements for experimental investigation of local plasma parameters. A novel threedimensional (3D) probe manipulator has been designed, implemented and successfully operated at VinETA.II. This work presents its design and performance for three-dimensional measurements of Vineta.II plasmas. Its design consists of three vertically stacked independent and mutually perpendicular linear motion stages which allow flexible positioning of diagnostic tools such as electrical and magnetic probes or optical diagnostics within the vacuum vessel. Its design features include a wide spatial coverage, sub-millimeter positioning accuracy and the capability to operate in a harsh environment under the influence of microwaves, radio-frequency waves and direct contact with plasma. Manipulator performance is assessed by measuring a volumetric distribution of plasma parameters by a B-dot probe. A typical discharge of the magnetic reconnection setup in VINETA.II with a pulse time of $\tau=600 \mu \mathrm{s}$ is chosen for this purpose. The azimuthal magnetic field distribution measured with the 3D manipulator agrees favorably with measurements obtained by the two-dimensional (2D) manipulator, used at VINETA.II as a standard reference diagnostic tool, thereby demonstrating its reliability and performance. A programmable stepper motor controller (TMCM-1110) that is operated remotely by a PC drives all possible features of the manipulator system.

Keywords: Overall mechanics design, Plasma diagnostics - probes

\footnotetext{
${ }^{1}$ Corresponding author

${ }^{2}$ Also at Technical University of Denmark

${ }^{3}$ Also at Institut für Physik, Ernst-Moritz-Arndt Universität Greifswald, Germany
} 


\section{Contents}

1 Introduction 1

2 Mechanical components 2

2.1 Z-Stage 2

2.2 X-Stage 3

2.3 Y-Stage 3

3 Control system 5

4 Application and Discussion $\quad 5$

5 Summary 17

\section{Introduction}

VINETA.II is a linear laboratory experiment developed for the study of driven magnetic guide field reconnection in low temperature plasmas [1,2]. Reconnection is driven by externally forcing magnetic field lines towards a central X-point, thereby inducing a current in a background plasma and consequently modifying the magnetic field topology. Magnetic reconnection is generally a three-dimensional(3D) phenomenon [4-7] that requires volumetric measurement of relevant plasma parameters. Its study requires a high spatial resolution, especially in the vicinity of the reconnecting current sheet. Flexible positioning of diagnostic probes at any position inside the vessel therefore offers many opportunities for detailed experimental investigation of reconnection. VINETA.II is equipped with one and two-dimensional manipulator systems that provide precise positioning in the azimuthal plane and along a radial cut, respectively.

Standard diagnostic probes available at VINETA include B-dot probes [8], Langmuir probes [9, 10]. The vacuum vessel is equipped with large ports that give access to the entire azimuthal crosssection of the plasma as well as several radial cuts through its center. Additionally, the vessel is large enough to accommodate a new low profile 3D positioning system entirely within the vessel without intersecting the plasma and or other in-vessel components. This manipulator system has been successfully developed and installed, allowing for free probe movement independent of port sizes and enabling truly 3D measurements. Installing the manipulator inside the vacuum vessel in the vicinity of the plasma poses significant design challenges that have been met by adhering to the following design and performance requirements:

- The design should be compact enough to introduce minimal plasma perturbation.

- All electrical components such as signal and power lines and motors should be shielded from microwaves or radio-frequency (rf) waves (used at VINETA for plasma production) to minimize the pickup noise. 
- The system should provide easy access for regular maintenance of moving parts.

- Accurate reconstruction of derived physical quantities such as the current density requires sub-millimeter accuracy in absolute positioning of diagnostics probes. The accuracy of both mechanical constructions and motor travel distances must meet these tolerances.

- Thermal load caused by microwave or rf power deposition on plastic and metallic components should not degrade system perfomance.

- Remote operation requires a sophisticated control system and user interface.

- Limit switches are required for all carriers to prevent the overrunning and jamming.

- Limit switches are required on all stages to prevent their jamming.

- Vacuum-to-air interfaces are required for all motor power and signal lines.

- The reconnection drive pulse in VINETA.II causes appreciable perturbation of the vessel ground potential and all in-vessel components. This electrical interference should be minimized.

These remainder of this paper addresses the realization of these requirements and showcases sample measurements performed with this system in the magnetic reconnection setup.

\section{Mechanical components}

The 3D manipulator setup is shown in Fig. 1. It consists of 3 vertically stacked independent and perpendicular linear stages. These stages determine the motion along the $z, x$ and $y$ axes and are correspondingly labeled in the figure. The size of the manipulator in each dimension is indicated in the figure by black arrows.

\subsection{Z-Stage}

The z-carrier, on which the two other stages are mounted, contains two linear bushings that enable motion along two round linear guide rails $16 \mathrm{~mm}$ in diameter. The carrier is also linked to a motordriven $\varnothing 14 \mathrm{~mm}$ thread bar by a ball screw. An ORIENTAL MOTOR PK266PDA stepper motor is used to drive the spindle, delivering a maximum torque of $3.5 \mathrm{Nm}$. The connection is made by a self-aligning coupling that provides heat expansion stress relief. The entire motor is screened from the $\mathrm{cw}$ microwave or rf radiation by a grid. The Z-carrier connects both to the rails and the thread bar, which can lead to immobilization due to inhomogenous thermal heat expansion stress from microwave or rf power absorption. The linear ball bearings are therefore connected to the carrier with a $0.5 \mathrm{~mm}$ clearance to relieve possible shear forces.

Limit switches prevent overrunning and jamming and mechanical crashes between the ZCarrier and the front or rear frames. These freely positionable switches are installed on both sides of the carrier and allow a maximum travel distance of $800 \mathrm{~mm}$. Conventional sealed industrial limit switches for use in harsh environments are used that are not vacuum compatible. In order to prevent pressure difference related malfunctions, small holes have been drilled in all limit switch housings. 


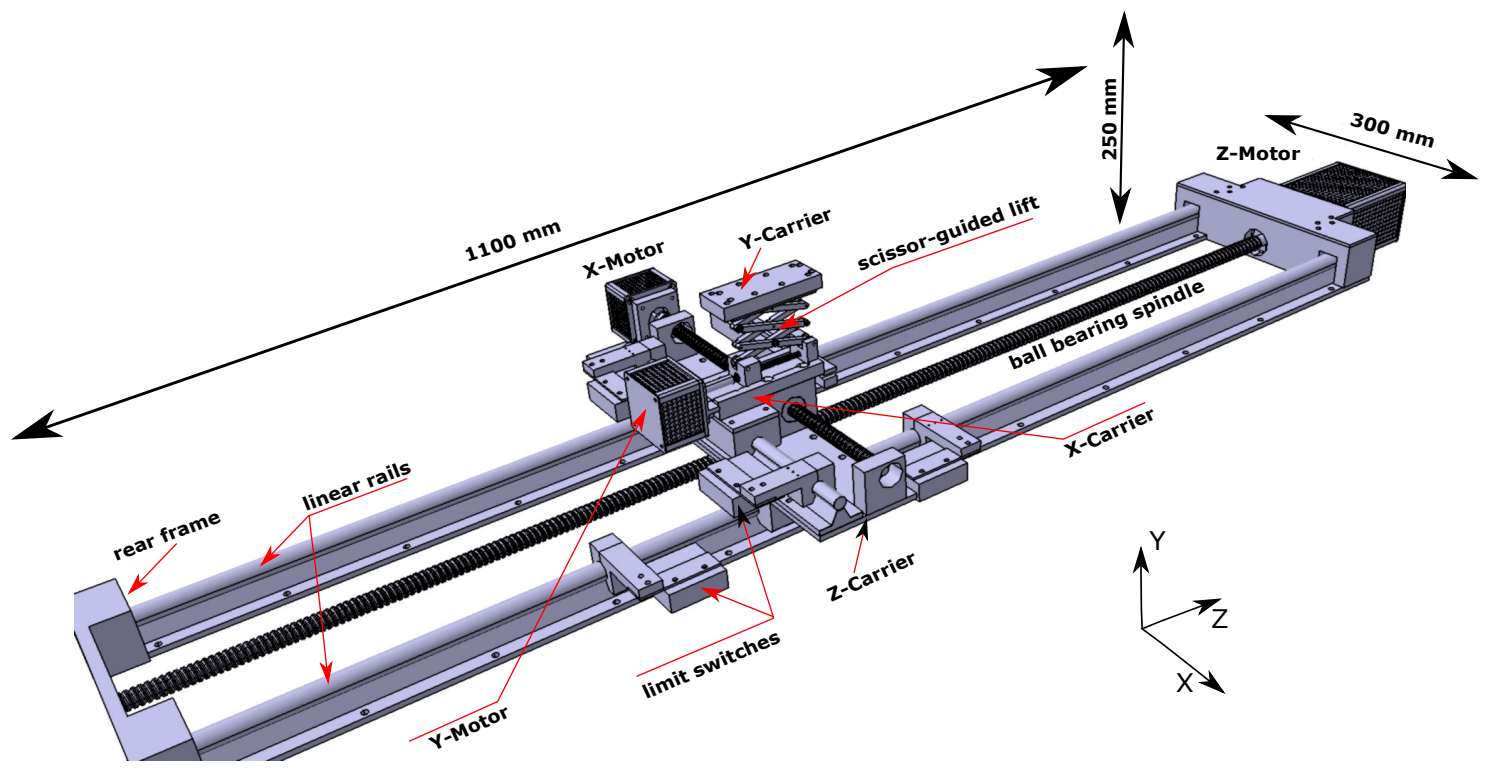

Figure 1. Isometric view of the three-dimensional manipulator. The spatial scale of the system is indicated by black arrows. The scale in the y direction corresponds to the retracted position of the y-stage.

\subsection{X-Stage}

The $\mathrm{x}$-stage is essentially a more compact version of the z-stage. This stage bears a much lighter load on its carrier, which allows for smaller support and drive mechanisms. In particular, a stepper motor with a torque of $0.2 \mathrm{Nm}$ is used (VEXTA PK243-01A), and the guide rail and spindle diameter are both $10 \mathrm{~mm}$. A single linear rail is sufficient here to support and guide the carrier motion together with the threaded bar as a second support. The maximum travel distance in this direction is $250 \mathrm{~mm}$.

\section{$2.3 \quad$ Y-Stage}

The y-stage is conceptually different from the previous two. Since this stage moves radially towards the plasma center, its design should be as compact as possible to minimize plasma perturbation by manipulator components. Fig. 2 shows the design of the scissor mechanism [3] used for this purpose. With this design, the probe carrier at its lowest position is still far enough $(130 \mathrm{~mm})$ from the plasma boundary.

Three consecutive scissors are extended by a stepper motor that turns a horizontal an M5 threaded bar. The same model of a motor is used here as the one for the $\mathrm{x}$-stage. Two miniature linear rails with a width of $6 \mathrm{~mm}$ guide both sides of the lift in order to stabilize sideways motion. A $4 \mathrm{~cm}$ long self-aligning coupling reduces the high degree of accuracy otherwise required for alignment of the drive motor shaft with the threaded rod. Miniature ball bearings are installed at all junctions between arms in order to reduce friction while keeping mechanical tolerances low.

Scissor mechanisms have a nonlinear dependence of their vertical displacement as a function of the horizontal base point displacement. Fig. 3 left shows an isosceles triangle corresponding to a single segment of the lift in which the sides $\mathrm{AB}$ and $\mathrm{BC}$ correspond to two arms of $l=32.5 \mathrm{~mm}$ 


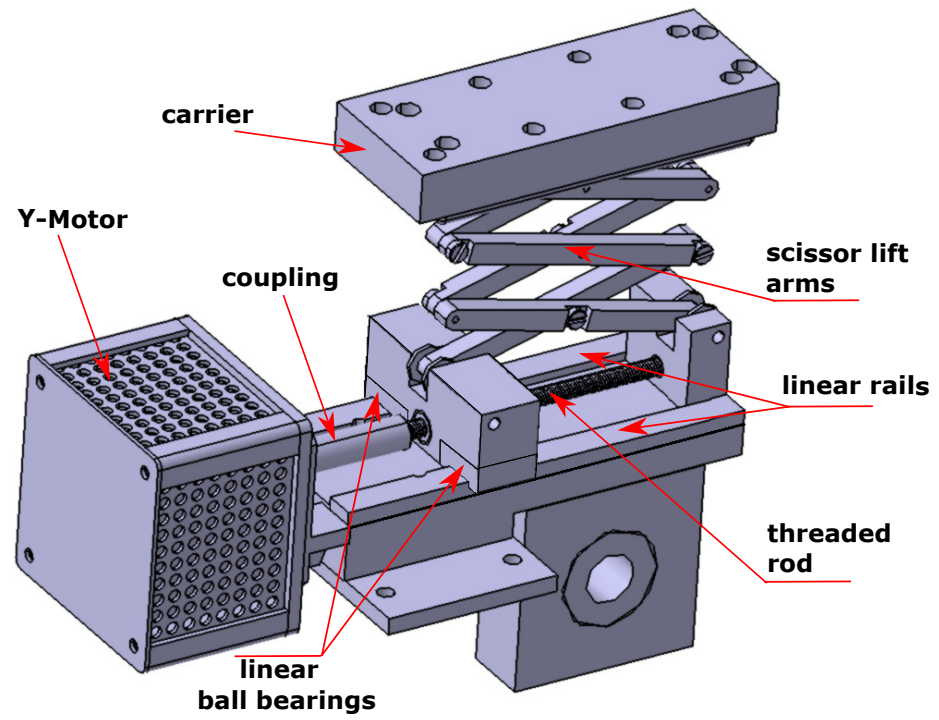

Figure 2. Isometric view of the y-stage.
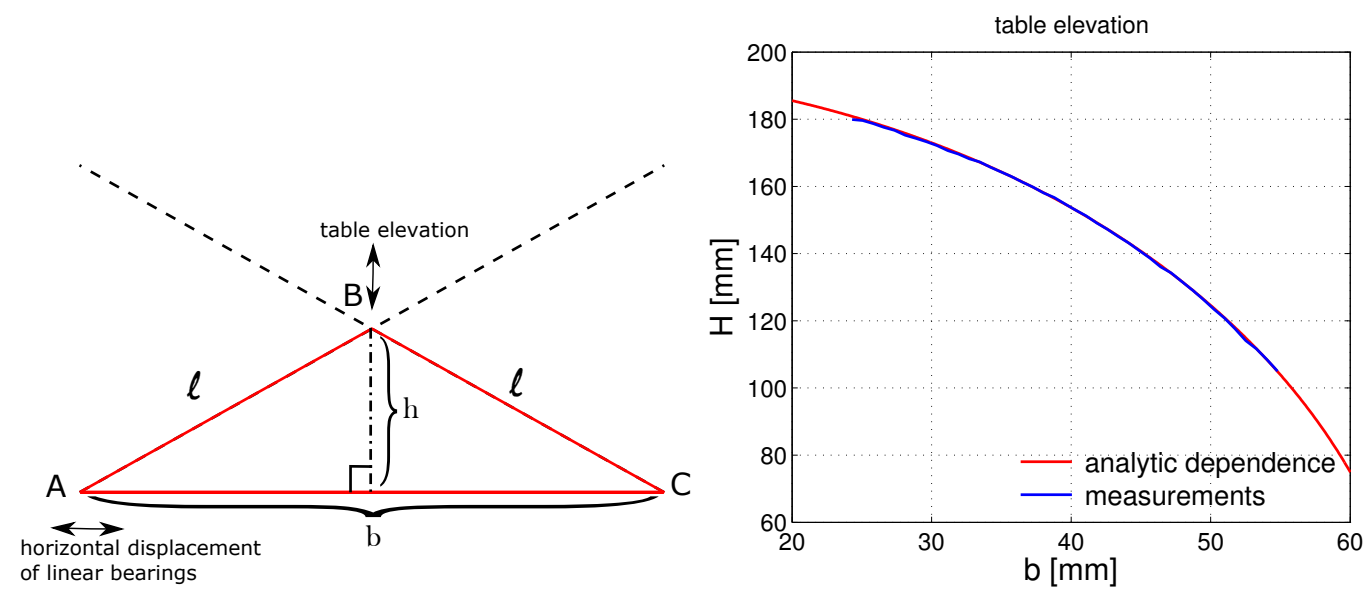

Figure 3. Left: Sketch illustrating a single segment of the scissor mechanism. Right: Elevation of the y-stage scissor mechanism vs. horizontal displacement of its base point. Red and blue lines indicate the analytic and experimentally measured dependencies, respectively.

length connected at point $\mathrm{B}$. The stage elevation $h$ as a function of the base length $b$ is then:

$$
h=\sqrt{l^{2}-\left(\frac{b}{2}\right)^{2}}
$$

The elevation of the entire mechanism is therefore $H=2 N h$ where $N=3$ corresponds to the number of consecutive scissors. Figure 3 right compares the analytical lift elevation derived from Eq.2.1 (red line) to the measured values (blue line). The match between these curves indicates overall stability of the lift design and implementation. The nonlinearity is clearly visible, and this dependence is used in data evaluation for conversion between the horizontal displacement input values and the actual elevation of the probe carrier. A significant mechanical disadvantage of scissor 
mechanisms is the considerable amount of force required to lift the scissor arm from a compressed position. In our setup, the angle between the base and arm must be at least $30^{\circ}$ for reliable operation in vacuum. This limits the maximum travel range to 76 out of the available $120 \mathrm{~mm}$.

During plasma operation, sputtered impurities are gradually deposited on manipulator components, thereby increasing the already heightened friction forces in vacuum on all moving parts. This applies to linear rails, threaded bars, ball screws and bushings, which therefore require periodic maintenance including cleaning and lubrication to prevent seizing. Additionally, a fraction of the microwave or rf power is partially absorbed by plastic and metallic components, which are therefore shielded by aluminium foil.

Electrical connections through the plasma vessel wall are made by vacuum-sealed D-sub connectors embedded in two CF63 vacuum flanges. These include power lines for all 3 motors, the 6 associated limit switch lines and 18 measurement signal cables. The motor power lines carry a modulated current of up to $2.5 \mathrm{~A}$ and are therefore routed through a separate flange from the measurement signals and take different paths in the vacuum vessel in order to prevent cross-talk. The signal cables are realized as 9 twisted pairs in order to reduce electromagnetic interference, especially from the reconnection drive conductors that are pulsed with a current of several $\mathrm{kA}$ on a microsecond timescale. Additionally, these large pulsed currents induce strong perturbations on the local ground potential with respect to the laboratory ground. The power and signal line shields are therefore insulated from the vessel walls and externally connected through the D-sub connectors. Further, the probe holder is insulated from the manipulator system itself to provide versatile grounding options.

\section{Control system}

All motors are controlled by TMCM-1110 universal stepper motor controller modules. The motion control program stored on these modules is written in the Trinamic Motion Control Language (TMCL). These programs take inputs both from the limit switches and carefully retract the stages on contact in order to prevent mechanical damage as well as from the user through the USB interface. User input is provided through LabView programs which integrate with a suite of other tools that set up the reconnection pulse sequence, trigger data acquisition and store measurement data. These programs set the positioning system to scan along a predefined equidistant grid (in one to three dimensions) with a given step size in any order of dimensions, or along a trajectory specified in an ASCII file. They also provide a user interface to set stage velocities, acceleration and calibration factors. This set of tools enables fully automated, reproducible 3D measurements of plasma parameters in the reconnection setup, which is crucial when dealing with a high number of spatial points.

\section{Application and Discussion}

To demonstrate the 3D capability of the developed system, exemplary measurement results from the reconnection setup are shown in Figure 4. Here, volumetric data taken in 5 planes with a spacing of $75 \mathrm{~mm}$ and recorded by a B-dot probe that measures the three magnetic field components $B_{x}, B_{y}$ and $B_{z}$ are shown. Sample reconstructed magnetic field lines are shown in blue. The local current 


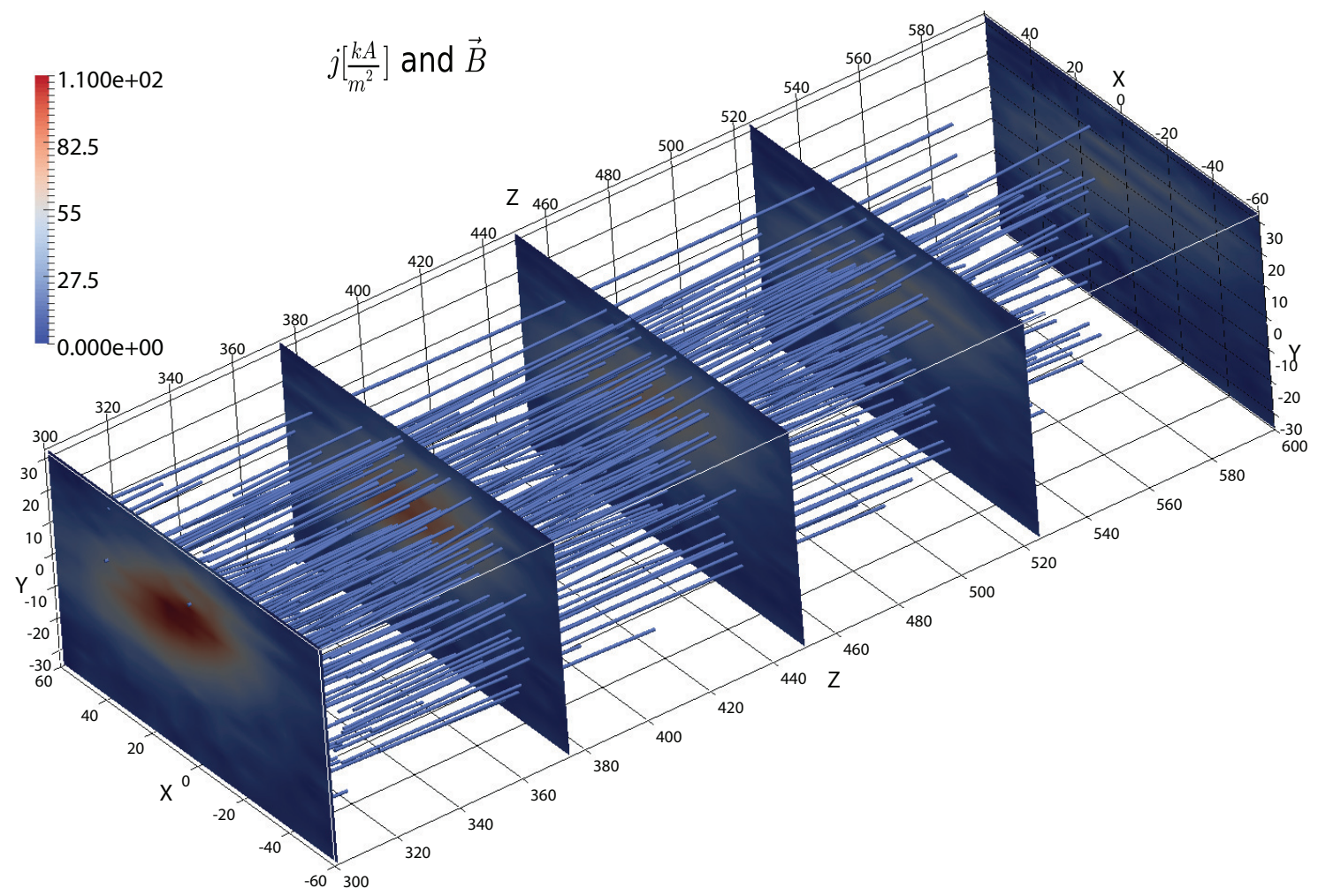

Figure 4. Volumetric data measured using the 3D manipulator using a B-dot probe in 5 azimuthal planes. Color plots denote the absolute current density value $j$ derived from the magnetic field components $B_{x}, B_{y}$ and $B_{z}$. Magnetic field lines are shown as a blue solid lines throughout the volume.
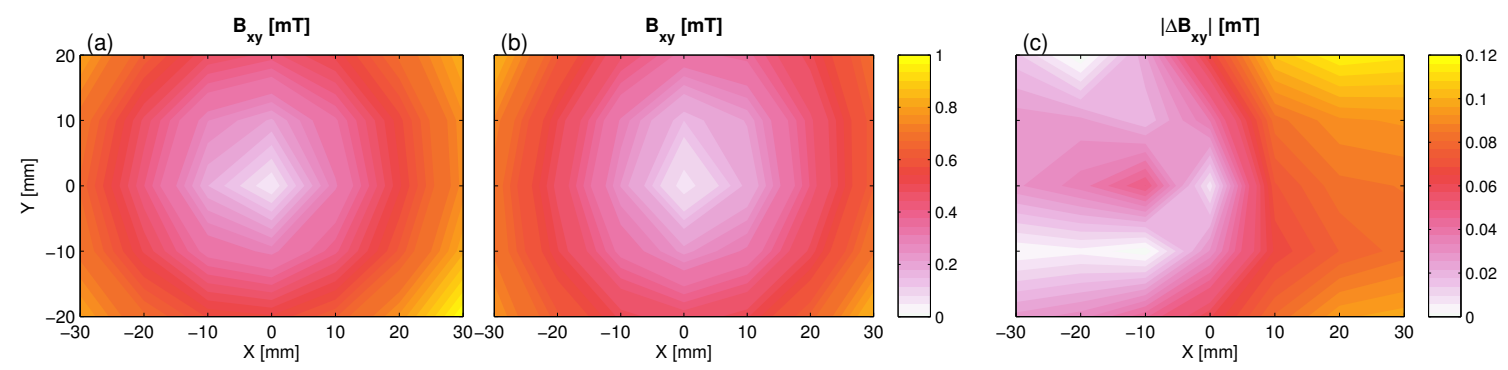

Figure 5. Comparison of the absolute azimuthal magnetic field distribution $B_{x y}(x, y)$ in the axial midplane. (a) and (b) are results from the 2D and 3D manipulators, respectively, and (c) is the absolute value of their difference.

density in each plane is calculated from $\mu_{0} \vec{j}+\mu_{0} \epsilon_{0} \partial \vec{E} / \partial t=\nabla \times \vec{B}$, where the contribution of the displacement current can be neglected to yield $\mu_{0} \vec{j} \approx \nabla \times \vec{B}$. The absolute value of $j$ is plotted in five azimuthal color plots, which show a 3D structure of the current sheet and demonstrate the new possibilities in characterizing magnetic reconnection at VINETA.II.

Commissioning of this new positioning tool requires a comprehensive assessment of its performance, which has been done by comparing to a two-dimensional positioning system of known accuracy as reference. This system is installed at the large radial port in the axial midplane of the 
vacuum vessel, giving access to a large part of its azimuthal cross-section. For this comparison, both systems have been equipped with calibrated B-dot probes which enables a direct comparison of the magnetic field distributions in the same measurement plane. The measurement results are shown in Figure 5, where the contour plots (a) and (b) show the azimuthal magnetic field distribution $B_{x y}(x, y)$ as measured by the 2D and 3D manipulator, respectively, and Figure (c) shows the absolute value of the difference between plots (a) and (b). These measurements have been made with a resolution of $1 \mathrm{~cm}$ so that the total number of measured points in $\mathrm{x}$ and $\mathrm{y}$ directions are 7 and 5, respectively. The consistency between both results with maximum error of about $15 \%$ in the top-right corner demonstrates the reliability of the developed hardware.

\section{Summary}

This work addresses the design and performance of a newly developed 3D manipulator for flexible position of plasma diagnsotic probes at the VINETA.II experiment, which is an essential tool for experimental investigations of magnetic reconnection. The presented design has several features beneficial for measuring plasma properties in laboratory experiments: 3D volumetric positioning over a wide spatial range, sub-millimeter positioning accuracy and the capability to operate in a harsh environment under the influence of microwaves, $\mathrm{rf}$ waves and under direct plasma exposure. It consists of three vertically stacked and mutually perpendicular linear stages that are driven by programmable stepper motors and remotely controlled through a PC.

Manipulator performance has been assessed by measuring a volumetric distribution of the magnetic field in a magnetic reconnection cycle. The consistency between in-plane magnetic field distributions $B_{x y}(x, y)$ measured by a reference 2D manipulator and the new system demonstrate the capability of the developed hardware.

The 3D output provided by the developed system opens new frontiers for full 3D characterization of magnetic reconnection and the associated current sheets.

\section{References}

[1] H. Bohlin, A. von Stechow, K. Rahbarnia, O. Grulke and T. Klinger VINETA II: A Linear Magnetic Reconnection Experiment, Rev. Sci. Instrum. 85 (2014) 023501

[2] A. von Stechow, O. Grulke and T. Klinger Experimental multiple-scale investigation of guide-field reconnection dynamics, 2016 Plasma Phys. Control. Fusion 58 (2016) 014016

[3] A. Hendrickson, Mechanical Design For the Stage, Elsevier, Oxford (2008).

[4] B. V. Somov, Plasma Astrophysics, Part 2, Chapter 5, Springer, New-York (2013).

[5] W. Gonzalez and E. Parker, Magnetic Reconnection, Concepts and Applications, Chapter 3, Springer, Heidelberg (2016).

[6] E. Priest and T. Forbes, Magnetic Reconnection, MHD Theory and Applications, Chapter 8, Cambridge University Press, Cambridge (2006).

[7] J. Birn and E. Priest, Reconnection of Magnetic Fields, Chapter 2, Cambridge University Press, Cambridge (2007).

[8] S. Ulrich, Electromagnetic Drift Waves, $\mathrm{PhD}$ Thesis, Ernst-Moritz-Arndt-Universität Greifswald (2010). 
[9] T. Windisch, Intermittent events and structure propagation in plasma turbulence, $\mathrm{PhD}$ Thesis, Ernst-Moritz-Arndt-Universität Greifswald (2007).

[10] I. Shesterikov and others, Measurements of Plasma Profiles Using a Fast Sweeping Langmuir Probe in the VINETA.II magnetic reconnection experiment, Journal of Instrumentation., submitted. 